TITRE: INFORMATION MUNICIPALE ET LECTURE CITOYENNE : HORS-NORMES ET NORMALITÉ DES PROCESSUS INTERPRÉTATIFS DANS DES TEXTES OUVERTS

Auteur(s): Karine Collette, Professeure agrégée, Département des lettres et communications, UniVERSITÉ DE SHERBROOKE, QUÉBEC, CANADA

PUBLICATION: ÉCRITS HORS-NORMES

PAGES: $140-153$

Directeurs: Agnès Steuckardt et Karine Collette

ÉdITEUR: LES ÉDITIONS DE L'UNIVERSITÉ DE SHERBROOKE, 2019

ISBN: 978-2-7622-0360-8

URI: HTTP://HDL.HANDLE.NET/11143/15578

DOI: HTTPS://DOI.ORG/10.17118/11143/15578 


\section{Information municipale et lecture citoyenne : hors-normes et normalité des processus interprétatifs dans des textes ouverts}

Karine Collette, Professeure agrégée Département des lettres et communications, Université de Sherbrooke, Québec, Canada

Résumé : L'information municipale est approchée du point de vue de l'interprétation empirique, en termes de lecture citoyenne, une reconstruction de sens d'extraits de procès-verbaux, avis publics ou encore communiqués, manifestant la participation discursive des lecteurs au sens des informations véhiculées. L'articulation à la problématique du hors-normes repose sur le repérage et l'identification de stratégies interprétatives qui révèlent, particulièrement selon le modèle de compréhension intégration de Kintsch et Van Dijk, une expression des connaissances, valeurs, expériences, logiques de pensée des lecteurs-compreneurs à la base de texte. Nos observations conduisent notamment à restituer aux lectures citoyennes de ces écrits professionnels, le caractère transitoire alloué aux textes littéraires hors-normes, car la part hors-normes des stratégies de reconstruction de sens relève d'une logique sociocognitive narrative où la dénomination des acteurs, des actions, de leurs motifs et incidences permettrait aux citoyens de questionner la pertinence sociopolitique des décisions. Où le hors-normes des discours interprétés s'inscrirait dans le genre et présagerait quelque changement possible au pallier de gestion politique le plus proche des citoyens.

Mots clefs : Information municipale ; lecture citoyenne ; processus d'interprétation ; interprétation horsnormes ; interprétation standard

Municipal information can be approached from the point of view of empirical interpretation, in terms of the citizen reader, a reconstruction of the meaning of the excerpts of minutes, public notices, or official municipal communication, that demonstrates the discursive participation of readers in the sense of information. Articulating the problematic of non-standard reading practices relies on spotting and identifying interpretative strategies that reveal, according to the model of comprehension integration proposed by Kintsch and Van Dijk, expressions of the readers knowledge, values, experiences, and views of the logic readers involved with the text. Our observations lead us to restore to the citizen reader of these professional texts the transitory character allocated to non-standard literary texts, because the non-standard part of the process of meaning reconstruction is based on a socio-cognitive narrative logic where the identity of the actors, their actions, their motives and their impacts would allow citizens to question the socio-political relevance of political decisions. The non-standard interpretation of these discourses would integrate into the ongoing institutional discourse and make visible any possible changes to the political decisions that are important to citizens.

Key words : Municipal information ; citizen reader ; interpretation process ; non-standard interpretation ; standard interpretation 
Si l'on aura toujours besoin des journaux, les pensées et interprétations qu'ils véhiculent seront prises avec de plus en plus de circonspection. Elles seront de moins en moins accueillies comme des informations pures, mais comme des éléments indicatifs et partiels pouvant servir à la construction d'une vision plus personnelle et plus indépendante. (Martin-Lagardette, 2005)

L'information municipale, abordée en tant qu'ensemble des écrits publics municipaux couramment publiés, s'inscrit ici dans le champ novateur de la rédactologie, soit l'étude des processus de rédaction et de réception des écrits dits professionnels, c'est-à-dire les textes rédigés par des professionnels de la rédaction (rédacteur administratif, technique) ou d'un domaine de spécialité, comme dans le cas d'une recommandation sanitaire émise par un épidémiologiste. L'étude dont il est question concerne particulièrement la connaissance - actuellement déficitaire, des manières dont les lecteurs peuvent interpréter ces écrits.

Traditionnellement, les manuels et guides francophones de rédaction administrative, technique et scientifique se basent sur les normes textuelles et stylistiques (plans de texte, tournures de phrases, etc.) réglementant l'écriture. Les facteurs de lisibilité d'un texte, la logique textuelle établie, la gestion des éléments linguistiques et relationnels, etc. ont été également exploités dans des projets de simplification du langage administratif en France (Collette et al., 2002), et au Québec, (Clerc (dir.), 2003). Toutefois, les écrits professionnels (textes administratifs, de presse, ici ceux de l'information municipale) sont quasiment ignorés sous l'angle de la reconstruction de sens par les récepteurs, au sens interprétatif et empirique.

Quand on s'interroge sur des textes (administratifs, de presse, etc.) qui ont une portée interprétative sociale voire politique; quand, pour questionner leur capacité à faire sens pour les lecteurs (intelligibilité des textes), on s'intéresse aux manières dont les lecteurs reconstruisent les sens, sur la base de leurs propres cadres de référence selon une conception interprétative empirique, les analyses ne visent pas l'évaluation de la lecture ni l'accomplissement de la procédure édictée, toutes deux relevant de perspectives plutôt normatives. Ici, il s'agira d'observer et tenter de saisir des manières de comprendre, au sens de " prendre avec », à savoir prendre le texte avec et dans le contexte de réception. Ces textes constituent un pan important de l'organisation sociétale et, en des termes participant de l'idéologie dominante, ils représentent une part non négligeable de l'économie de l'écrit (emplois rédactionnels, communication et archives publiques...), contribuant fortement à l'actualisation de la littératie voire de la vie citoyenne, du moins en ce qui concerne les connaissances liées aux règles et au fonctionnement de la cité.

Nous définirons la littératie citoyenne comme l'ensemble des traces discursives liées à la capacité à comprendre pour réfléchir discursivement à l'organisation et aux valeurs de la société et donc prendre part à la vie sociopolitique. La littératie citoyenne est fondée sur des perspectives de développement à la fois personnelles, sociales et politiques. Ainsi, cette question de la construction de sens des écrits publics de la ville dans une perspective interprétative, aussi critique et réflexive, trouve-t-elle sa pertinence sociale les déclarations publiques des élus, les évènements constituent autant d'informations à interpréter pour accomplir la participation citoyenne, d'une manière la plus cohérente possible aux valeurs et conditions de vie des individus composant la collectivité.

Dans le cadre de réflexion sur les écrits hors-normes, les questions guidant notre propos se formulent comme suit: comment le contexte de réception - hors des normes du cadre de production des écrits municipaux à l'étude -, s'avère-t-il constitutif des reconstructions de sens empiriques par les citoyens-lecteurs? Par quelles manières de reconstruire le sens, par quelles stratégies de compréhension-interprétation, ce hors-normes, postulé du point de vue d'une conception centrée sur le pôle production - où le cadre de 
production imposerait son contexte à la reconstruction de sens -, par quels processus de compréhension-interprétation donc, et selon quelles modalités ce hors-cadre est-il intrinsèquement lié au genre des écrits publics municipaux, si l'on postule qu'il existe encore une lecture citoyenne, active par définition?

\section{Considérations théoriques}

Les activités de compréhension-interprétation des écrits dépendent de paramètres linguistiques (mots, syntaxes, etc.) et d'autres, non-linguistiques, soit les connaissances préalables des lecteurs, leurs représentations (i.e. les croyances, la culture, les idéologies), leurs projets de lecture, leurs conditions sociales, affectives et relationnelles. Ces paramètres non-linguistiques se combinent aux éléments linguistiques pour façonner la compréhension-interprétation. Ils sont variablement inscrits dans des modèles ou théories largement reconnus, comme le modèle de situation (Van Dijk et Kintsch, 1983), la logique naturelle (Borel, Grize et Miéville, 1982, 1992 ; Grize, 1990, 2003) ou encore la théorie de la pertinence selon Sperber et Wilson (1989). Le modèle de Kintsch souligne que la compréhension s'effectue en fonction de la représentation qu'on se fait de la situation exposée dans un texte ou message. Un lien important entre expérience de vie, mémoire et compréhension est ainsi établi, lequel lien présage la possibilité d'émergence du hors-normes dans la lecture-interprétation de textes ouverts, puisque les contextes de production et de réception sont disjoints et dans la mesure où le hors-normes se détermine possiblement au regard de paramètres sociologiques Par ailleurs, Grize pose que les pôles de production et de réception ne peuvent jamais être identiques dans la communication humaine et que les images qu'un pôle se fait de l'autre, de ses connaissances, de ses intentions, participent à la production et à la compréhension des messages. Sperber et Wilson réaffirment que c'est le destinataire (ici le lecteur) qui (re)construit la pertinence d'un texte, au regard de sa propre situation. II résulte de ces théories et modèles que le lecteur, « compreneur » et interprétant, investit des connaissances, reconstruit le sens du texte avec des éléments d'ordre personnel (ses propres connaissances, expériences, ses attentes et perceptions) et cognitif (ses capacités à comprendre, ses stratégies de lecture et de compréhension) mais encore d'ordre social (ses repères culturels, croyances et opinions de référence, ses représentations) et communicationnel (qualité de la relation, conditions de l'interaction texte-lecteur, identification des intentions). Nos analyses d'interprétations de lettres administratives (2004) ou d'articles de journaux (2007) montrent aussi que les interprétations tissent un maillage discursif et sociocognitif très serré entre des éléments tirés du texte et ceux dont on ne tient généralement pas compte parce qu'ils sont colportés par le lecteur (les représentations, connaissances antérieures, repères idéologiques, valeurs, etc.) (Collette, 2004, 2005, 2007, 2009).

Centrées sur la norme textuelle ou l'intention de communication selon le mandat, les travaux en rédaction professionnelle se limitent pourtant généralement à postuler un lecteur largement coopérant à l'égard du pôle production, sinon déficitaire. Bref, un lecteur qui épouserait la logique instituée via le texte, sans considération des décalages entre les sphères de production et de réception, sauf en termes de compétences techniques.

Or, la réception d'une grande part des écrits professionnels (articles de presse, écrits administratifs « externes ») se réalise en dehors des champs de leur production (service administratif, milieu journalistique). À la suite de la théorie de Halbwachs (1952), après celle de Lahire (2001) et selon nos propres recherches (2004, 2005, 2007, 2008, 2009), la conception même de la compréhension d'un texte se trouve modifiée dès lors que le texte est produit dans un contexte (ici professionnel, juridico-administratif) et lu dans un autre (ici citoyen). La transposition d'un texte, d'un contexte à un autre, en modifie potentiellement le sens. En 
écho à la conception de Grize, le hors-normes, observé au plan empirique de la reconstruction de sens, ellemême discours, s'immiscerait naturellement entre ces deux sphères distinctes de production et de réception des discours, mises en relation par le truchement de la communication. La communication à l'œuvre, entre un pôle de production d'un discours fortement ritualisé et technique, et un pôle de réception externe à ce cadre (exogène), suppose l'émergence d'un discours intermédiaire, fort probablement hors-normes du point de vue de la production. Les caractéristiques intrinsèques du hors-normes ici à l'étude laisseraient entrevoir son potentiel de changement communicationnel, sinon sociopolitique : le hors-normes repéré dans les manières de reconstruire le sens de l'actualité municipale offre un espace discursif d'actualisation sociopolitique de la lecture citoyenne. En ce sens, le hors-normes jouirait du caractère transitoire reconnu au hors-normes en littérature et rappelé ici par S. Bikialo. Perturbations, altérations du texte source passées sous silence dans la réception des écrits professionnels, alors qu'elles font l'objet de valorisation en littérature, les manifestations du hors-normes, dans la reconstruction de sens des écrits qui régulent, tracent peut-être la voie de changements communicationnels des institutions, prémisses de modifications sociopolitiques dans nos démocraties contemporaines. Les observations invitent à un enthousiasme moindre.

\section{Éléments de méthodologie}

L'étude repose sur un corpus hétérogène (Bonnafous et Temmar, 2007) contenant: a) un recueil d'avis publics (textes très courts, aux formes récurrentes et publiés dans la presse locale), b) un recueil de procès-verbaux des réunions publiques du conseil municipal (textes longs, distribués lors des réunions publiques du conseil municipal et publiés sur le site de la ville, c) un recueil de reconstructions de sens des avis publics et des parties sélectionnées de procès-verbaux (car les PV sont des documents trop longs pour être soumis dans leur entièreté à des interprétations), d) un ensemble « témoin » d'articles de journaux qui couvrent l'actualité municipale (un journaliste de la presse locale est attitré aux affaires municipales, d'autres textes présentent des allocutions ou déclarations des représentants de la ville (maire et conseillers municipaux), d'autres articles thématiques paraissent aussi ponctuellement). La pertinence de ce $4^{e}$ ensemble tient aux résultats obtenus dans l'étude préliminaire de ladite recherche, concernant l'intertextualité et l'interdiscursivité des écrits autour du conseil municipal. Cette étude initiait l'analyse des relations entre trois genres d'écrits publics (PV, avis publics et articles de journaux), collectés sur une période de 22 semaines (20072008). Les premiers constats (Collette, 2008) indiquent que certaines rubriques des procès-verbaux (urbanisation, matières résiduelles et environnement. .) sont régulièrement relayées dans les articles de journaux. Pourtant, les procès-verbaux ne figurent jamais au titre de sources documentaires dans les articles étudiés; les journalistes réfèrent à des documents de travail des comités (plan d'urbanisation, plan directeur du projet, etc.) non publiés, auxquels ils accèdent parfois mais dont les lecteurs ne disposent pas. Par ailleurs, comparé au traitement administratif de l'information dans les PV et avis publics, le traitement qui est fait de l'actualité municipale dans les journaux est d'ordre politique: décisions commentées selon les orientations politiques des conseillers (rhétorique), prise en charge des décisions, personnalisation, polémiques...

Pour comprendre les manières dont les sens sont reconstruits, il faut tenter de saisir le parcours interprétatif effectué par chaque locuteur, sur chaque texte : repérer les éléments issus - implicitement ou explicitement - du texte lu, identifier les sources et les formes des reprises, emprunts, reformulations, identifier la hiérarchie informationnelle reconstruite par rapport à celle qui était proposée (repérer les éléments sélectionnés ainsi que leur place dans la configuration informationnelle, ceux qui disparaissent, etc., identifier les relations établies à d'autres textes (comme par exemple les articles de journaux), répertorier les 
barrières interprétatives, les dérives aussi parfois. L'exercice analytique du parcours interprétatif individuel (Collette, 2005) est nécessaire pour décrire finalement les différentes manières dont le sens peut se reconstruire. Au stade interprétatif des résultats, le concept de logique sociocognitive que nous empruntons à Windisch $(1982,1985,1990)$ prolonge l'analyse dans une perspective sociale, pour rendre compte de ce qui induit ou incite les interprétations du point de vue des repères sociaux. Entendues comme une construction d'opérations discursives, les logiques sociocognitives impliquent que les manières d'agencer les éléments des discours sont une trace de l'activité des paramètres contextuels sur le sens.

À partir de différents modèles et repères théoriques en compréhension et interprétation des textes, un cadre d'analyse avait été créé afin d'identifier différentes catégories de stratégies de compréhension : traces des stratégies de compréhension globale, traces des stratégies d'intertextualité, traces des stratégies de recours à la mémoire, traces des stratégies réflexives et critiques, traces des stratégies inférentielles et les passages concernant l'enquêté. Chacune de ces stratégies est elle-même subdivisée en différentes catégories.

Les résultats présentés s'appuient sur le modèle de construction-intégration de Kintsch et Van Dijk (1988 et suiv.). Ledit modèle vise une représentation mentale cohérente de ce qui est dit par le texte. Nous rappellerons que les auteurs distinguent deux types d'activités psychologiques. Particulièrement dédié aux activités considérées de bas-niveaux, le premier type d’activités assure la représentation du contenu du texte, sur trois niveaux:

a. Intrant linguistique: déchiffrement des mots et traitement lexical;

b. Niveau sémantique, construction de la microstructure assurant la cohérence locale (série de propositions issues des indices textuels et des connaissances du lecteur);

c. Construction de la macrostructure assurant la cohérence globale (reconfiguration informationnelle par hiérarchisation, condensation ou réorganisation).

Le second type d'activités vise l'intégration de la représentation du contenu du texte à la base de connaissances du lecteur. La représentation qui en résulte constitue un type de modèle de situation, une représentation mentale de la situation décrite par le texte. Cette représentation cognitive comprend des évènements, des actions et des individus liés à la situation qu'évoque le texte. Le modèle de situation incorpore des expériences préalables, des bases de texte antérieures, des particularisations de connaissances plus générales (Tapeiro, 1992). Une base de textes est constituée par la sélection, la modification et le réarrangement de concepts issus du texte et des connaissances du lecteur, lequel peut reconnaitre une situation familière : la compréhension sera alors une mise à jour d'un modèle de situation issu de la base de connaissances. Ce sont les connexions élaborées entre les connaissances du lecteur et celles issues du texte, la flexibilité cognitive et la force des connexions à l'œuvre, qui déterminent les capacités de compréhension des lecteurs. Le modèle de situation peut incorporer les expériences antérieures du lecteur et les représentations textuelles précédentes concernant des situations identiques ou similaires, ainsi que des éléments de connaissances plus générales concernant ces situations. La représentation correspondant au modèle de situation constituerait la trace la plus durable en mémoire. Ce sont alors les activités de type top-down qui caractérisent particulièrement le modèle de situation : la relation entre le signifiant et le signifié; la reconstruction des implicites; les inférences ; le contexte (situation pragmatique etc.). 


\section{Analyses}

\section{Six catégories de processus interprétatifs}

Dans les discours de reconstructions de sens par les lecteurs, nous avons identifié selon une démarche inductive, six catégories de processus inspirés de la base de texte et/ou du modèle de situation.

\section{Traces de processus interprétatifs et de compréhension globale}

Qui réfèrent à la base de texte comme par exemple le rappel, spontané et en priorité, d'éléments informatifs du texte lu ; la reformulation des éléments considérés comme prioritaires; la citation d’éléments qui retiennent le plus l'attention; les éléments liés au protocole, le cas échéant. On comptera aussi parmi ces stratégies l'expression d'une incompréhension, les questionnements relatifs à la recherche d'informations dans le texte ainsi qu'à la compréhension des contenus [ça veut dire quoi?; Je ne sais pas; Je ne comprends pas; où sont ces informations ?], les questionnements sur les valeurs rhétoriques des énoncés ou des éléments du texte (en termes de droit, d'obligation, etc.). D'autres processus de cette catégorie renvoient conjointement à la base de texte et au modèle de situation. Par exemple, les hypothèses de signification et les élucidations des «failles », des manques ou des déficits informationnels du texte, dans le texte. Les cas où le lecteur résout des questions informationnelles liées aux implicites du texte, ou lorsque l'interprétation en cours oscille entre questionnements, hypothèses de signification et conclusion interprétative.

Les catégories suivantes sont essentiellement comblées par des processus référant au modèle de situation, donc à des activités de lecture de haut niveau, relevant très clairement de l'interprétation des textes.

\section{Traces de processus d'intertextualité.}

Il s'agit ici de mentions référant à d'autres textes (ordre du jour, procès-verbal, etc.), à d'autres discours (radio-télévisés, rumeur politique ou sociale, etc.). Y compris lorsqu'en cas de questionnement, le lecteur évoque un autre discours qui pourrait, par exemple l'aider à clarifier une information : «Un urbaniste pourrait m'aider».

\section{Traces de processus de recours à la mémoire.}

Lorsque le lecteur énonce des connaissances déclaratives déjà-là, de type ceci signifie cela, lorsqu'il mobilise son savoir, sa connaissance pour raconter ce qu'il comprend du texte. Les bagages d'ordres technique, théorique et pratique sont constitutifs de ce processus lorsqu'ils sont convoqués explicitement pour raconter le sens du texte. L'expérience et le vécu des enquêtés, plus ou moins en décalage avec le texte, les exemples présentés par l'enquêté pour expliquer ce qu'il comprend.

\section{Traces des processus réflexifs et critiques.}

Celles-ci incluent les questionnements et développements relatifs au fonctionnement du système municipal, à l'organisation des processus décisionnels de la ville, des institutions, etc. Les jugements sur le système, tant positifs que négatifs du système institutionnel et politique, les revendications personnelles, passages relatifs à des critiques, questionnements portant sur la valeur ou la fonction du texte dans le système ou l'organisation des processus décisionnels. 


\section{Traces des processus inférentiels.}

Celles-ci concernent essentiellement des conclusions ou hypothèses interprétatives qui surviennent à la lecture du texte mais vont au-delà de ce que dit le texte (extrapolation), lorsque le lecteur « sort » du texte et va plus loin dans sa compréhension. Lorsqu'il met en œuvre une lecture active, au sens bakhtinien du terme. D’autres traces de processus inférentiels se rapportent plus particulièrement à la base de texte, dans le sens où le lecteur exprime son rapport au texte, en termes, par exemple, de dévalorisation (de ses compétences pour lire le texte), de démotivation. Lorsque ce dernier précise qu'il n'a pas d'intérêt pour le texte, que ses connaissances ne lui permettent pas de le comprendre. Ou encore lorsqu'il parle de lui, de sa propre expérience de lecteur ou de citoyen, en décalage avec l'objet du texte, en cours de lecture.

Nous présentons ci-dessous le résultat d'une analyse de 21 lectures d'un avis public « Règlement n 1-72 », du point de vue des traces de processus de compréhension-interprétations dominants répertoriés.

\section{Processus, tels que mis en œuvre}

Lorsque les enquêtés formulent ce qu'ils comprennent du texte, le processus interprétatif et de compréhension globale apparaît nettement dominant (32 occurrences). Notons que, régulièrement, les lecteurs utilisaient le même processus à plusieurs reprises dans leur réponse. Les chiffres présentés sous le terme d'« occurrences » regroupent les apparitions d'une même sous-catégorie de processus chez un enquêté. Autrement dit, si un usager a recouru trois fois au même processus interprétatif et de compréhension globale par récupération d'éléments provenant du texte, nous n'avons comptabilisé qu'une seule occurrence.

Le processus interprétatif et de compréhension globale réfère donc à la base de texte, rappelle spontanément et en priorité des éléments informatifs du texte lu ; les reformule; cite des éléments. Treize enquêtés ont utilisé celui-ci comme première stratégie de réponse, c'est-à-dire en première position. De ces 13 enquêtés, 5 l'ont utilisé seul pour répondre à la question. Dans les autres cas, il est par contre difficile d'identifier une constance dans le processus utilisé en deuxième position.

La récupération d'éléments provenant du texte concerne 19 de ces 32 occurrences totales. En ce qui concerne les autres aspects de cette stratégie, l'émission d'une hypothèse de signification ou élucidation de «failles » du texte totalise 8 occurrences, tandis que l'expression d'une incompréhension est décomptée 4 fois.

En deuxième position, les processus réflexifs et critiques comptent le plus grand nombre d'occurrences (13) après la précédente. Dans l'ordre d'importance : 4 enquêtés ont utilisé celle par critiques textuelles et rédactionnelles ; 1 enquêté, celle par questionnements et développements relatifs au fonctionnement du système ou à l'organisation des processus décisionnels; et unenquêté, celle par critiques du système institutionnel et politique. On observe 8 occurrences de cette stratégie, par des traces de critiques textuelles et rédactionnelles, trois par des questionnements et développements relatifs au fonctionnement du système ou de l'organisation des processus décisionnels, et deux enfin par des critiques du système institutionnel et politique.

Pas de constance du premier processus utilisé par les sept autres enquêtés. Trois enquêtés ont choisi un processus réflexif et critique : un enquêté, celui par questionnements et développements relatifs au fonctionnement du système ou à l'organisation des processus décisionnels, et deux enquêtés, celui par 
critiques textuelles et rédactionnelles. Pour les deux derniers enquêtés, cette stratégie a été utilisée seule dans un cas; dans l'autre cas, elle a été suivie d'une stratégie interprétative et de compréhension par expression d'une incompréhension (les deux seules stratégies utilisées dans la réponse).

Les quatre autres enquêtés ont tous utilisé un processus différent en première position, soit une stratégie interprétative et de compréhension par expression d'une incompréhension, une stratégie de recours à la mémoire par connaissances déclaratives préalables, une stratégie d'intertextualité et une stratégie inférentielle.

\section{Éléments thématiques sur lesquels s'articulaient les processus}

\section{Processus interprétatif et de compréhension globale}

Par récupération d'éléments provenant du texte (19):

Dans l'ensemble, 19 enquêtés sur 21 ont utilisé ce processus pour répondre à la reconstruction de sens du texte, peu importe la position de la stratégie dans la réponse. De ces 19 enquêtés, 14 ont entamé leur réponse par celui-ci. Le plus souvent (10 fois si on se permet de sauter une phrase ou deux dans certains cas), les enquêtés reprenaient le contenu du deuxième paragraphe du texte, en le reformulant ou en le citant, soit «QUE lors d'une séance régulière tenue le 20 décembre 2010, le conseil municipal de la Ville de Sherbrooke a adopté le règlement no 1-72 modifiant le règlement no 1 de la Ville de Sherbrooke de façon à [...] 》('italique correspond au segment qui revenait généralement dans la réponse).

Suivaient habituellement dans les réponses les éléments énoncés dans les tirets (sous le deuxième paragraphe) assorti d'un commentaire sur le défaut de cohérence entre ces éléments. Cette dernière observation des enquêtés était, le plus souvent, ce qui les amenait à utiliser d'autres stratégies (dans 13 cas), après le processus de compréhension globale par récupération d'éléments provenant du texte. Aucun enquêté n'a parlé de tous les tirets dans sa réponse. Dans le cas de la stratégie par récupération d'éléments provenant du texte, les enquêtés citaient soit des tirets en exemples, soit ceux qui les concernaient ou qui leur semblaient les plus simples à comprendre.

Par expression d'une incompréhension (4):

Le processus interprétatif et de compréhension par l'expression d'une incompréhension a été relevé trois fois pour la question des tirets. L'interrogation portait ultimement dans ce cas sur la valeur de certains énoncés de modification du règlement. Par exemple, l'enquêté 109 citait les énoncés qui lui posaient problème :

Mais je prends le premier : « modifier les dispositions relatives à la délégation du pouvoir d'autoriser des dépenses et de passer des contrats afin de prévoir différentes règles concernant les comités de sélection ». On n’a pas compris qui ça touche. «[À] la délégation du pouvoir ». C'est qui cette délégation-là ? On n'a absolument rien compris. On ne sait pas pourquoi c'est là. Qu'est-ce qu'ils vont modifier : on ne sait pas. [...] Même chose pour le troisième : « prévoir les dispositions applicables à la bibliothèque du secteur de Rock Forest ». Quelles dispositions applicables? [...] On ne sait pas pourquoi ils révisent par contre... [...]. 
L'autre enquêté ayant exprimé une incompréhension l'a fait relativement à l'avant-dernier «QUE » du texte, où des précisions sur certains des articles du règlement no 1-72 sont ajoutées et où des numéros d'articles sont pour la première fois introduits dans le texte. L'enquêté s'est demandé d'où provenaient ces numéros d'articles.

Par émission d'une hypothèse de signification ou par élucidation de « failles » du texte (8) :

Ce processus est manifesté pour différents segments du texte et apparaît majoritairement pour un seul segment. Deux enquêtés émettent des hypothèses quant à la nature des numéros d'articles introduits à l'avant-dernier «QUE » du texte. Une personne y va d'une hypothèse sur le sens du mot « abattis », utilisé au $12^{\mathrm{e}}$ tiret. Une autre personne émet une hypothèse sur les raisons possibles du retrait des feux de circulation à une intersection (5e tiret). Trois enquêtés interprètent la nature du règlement comme telle : respectivement, le règlement no 1-72 serait « un paquet de petites lois »; les modifications se feraient à des niveaux différents, selon différentes interventions dans la ville; et les mesures seraient officielles, le citoyen pourrait consulter le règlement. Un dernier enquêté soutient que l'avis public serait le résumé de quelque chose qu'il aurait déjà pu lire plus tôt dans l'entrevue, par exemple dans un procès-verbal. Notons encore que les autres processus n'ont pas été activés de façon significative et que 21 enquêtés ont utilisé de 1 à 3 stratégies pour exprimer leur compréhension du texte.

\section{Conclusion des analyses}

Ces observations nous permettent de tirer quelques enseignements des processus stratégies à l'œuvre dans la lecture-interprétation de cet avis-public:

Que le nombre de stratégies utilisées par les lecteurs oscille entre 1 et 3 tend à confirmer d'autres études concluant sur l'intérêt de développer un panel de processus et stratégies variés pour chaque lecteur, afin d'augmenter globalement les compétences lectorales.

Que les stratégies les plus couramment utilisées relèvent prioritairement de la base de texte (stratégie interprétative et de compréhension globale par récupération d'éléments du texte), qui plus est en première position, indique que le contexte de réception n'interfère pas initialement dans la reconstruction de sens des textes soumis à la lecture, qu'il n'y aurait pas dévoiement du sens proposé par le texte, du point de vue de la réception.

En revanche, les modes de rappel des éléments récupérés dans le texte, à savoir essentiellement des citations concernant les contenus exposés via les tirets dans le texte, nous incitent à souligner à ces endroits un déficit de traces d'appropriation du sens : les lecteurs ne semblent guère en mesure de reformuler les contenus essentiels du texte dans leurs propres mots : distance contextuelle et distance discursive sont alors à interroger du point de vue de la réception de ces textes. Car, à l'opposé de l'hypothèse concernant la contamination d'un contexte (production) par l'autre (réception) pour reconstruire le sens des textes, une réception qui saurait essentiellement citer ne présente guère d'atout au plan interprétatif. Notre démarche étant discursive, nous nous garderons ici de conclure sur un déficit de compétence lectorale des enquêtés et solliciterons de nouveau les observations concernant les thèmes sur lesquels s'appliquent les stratégies pour éclairer un peu la nature du problème. 
La processus majoritaire (par récupération d'éléments du texte) est suivi de près par celui qui exprime une incompréhension. L'enchaînement de ces deux stratégies s'effectue dans la même catégorie de la base de texte, mais soulève la question de la perméabilité du texte par les lecteurs. Les réponses aux questions exprimées leur permettraient une appropriation des contenus publiés. Ici, la question des contextes confère une résonnance particulière aux stratégies mises en œuvre, en termes de caractéristiques des logiques sociocognitives de la réception, versus celles de la production : pour que les contenus de l'avis public fassent sens, les lecteurs appellent à une désignation des acteurs et détails des éléments situationnels, permettant de contextualiser les énoncés, dans le sens d'une représentation possible. Ainsi, la demande des lecteurs concerne la dénomination d'acteurs non-désignés, le détail de modifications ou dispositions annoncées, les motifs pour lesquels des changements sont prévus.

\section{Discussion}

En matière de compréhension-interprétation des avis publics, si tant est que ces genres soient encore voués à quelque interprétation qui soit, force est de constater que les logiques sociocognitives à l'œuvre du côté de la réception agissent de sorte à façonner la représentation offerte au lecteur, selon certains aspects du cadre contextuel de ce dernier. Ces lectures sont-elles pour autant hors-normes ? Les éléments sur lesquels reposent ces incidences contextuelles ne transgressent pas radicalement, ni ne disqualifient les propos du texte proposé. La quête poursuivie par les lecteurs in situ, via leurs questions, s'apparente nettement à une démarche de précision des composantes (agents, conditions et évènements) de la représentation du texte, dans une perspective narrative. La distance discursive entre le langage administratif municipal - même publié - et les discours de reconstruction de sens trouve bel et bien un écho du point de vue de l'ancrage des logiques sociocognitives : où les modalités non-agentives et exemptes de précisions causales, sans mention exacte mais seulement générique des actes, et sans propension réflexive caractérisent le discours administratif municipal, la lecture-citoyenne active demande à instaurer les aspects de la discursivité narrative et réflexive, qui rendraient le discours passablement plus compréhensible aux lecteurs situés hors des cadres de la production. C'est qu'il faudrait au lecteur-citoyen des noms d'acteurs, des actions désignées et représentables, des motifs concernant les ressorts des décisions, pour être capable de se construire un scénario narratif et une scène idéationnelle qui permettent le jugement, dit éclairé, sur les informations décisionnelles publiées. Nous proposons, en guise d'ouverture, de considérer cette particularité lectorale sous la lunette des genres : les arrêts et détours des lecteurs, leurs quêtes de reconstruction narrative et réflexive, hâtivement qualifiables de hors-normes au regard des incidences lectorales écaillant le texte d'origine, seraient alors manifestation des caractéristiques dynamiques du genre, en action. Sortes de boucles réflexives coïncidentes aux logiques sociocognitives et discursivement marquées par le genre et par un ethos collectif de réception : citoyen-lecteur, pour qui le sens passe par la logique narrative et la rationalité explicite et causale des actions.

Hors-normes, donc, les reconstructions de sens de ces lectures? Hors des cadres de la production scripturale instruisant la norme, hors de sa position institutionnelle, certes. Mais normale (au sens quantitatif et probabiliste), du point de vue de la fréquence des processus identifiés et de leur position prioritaire dans le panel des quelques stratégies ici employées. Quand la norme socialement dominante se heurte au normal sur le plan des stratégies spontanément mises en œuvre, lesquelles se trouvent à être ici cautionnées du point de vue théorique, les rapports entre institutions administratives (politiques locales) et citoyens mé- 
ritent réflexion. La tension sociologique, de l'imposition d'une norme et de la résistance qui y fait face se manifeste discursivement, entre logique proposée et processus de reconstruction de sens.

Loin de faillir à sa tâche interprétative, le lecteur-citoyen, par ses apparents détours interprétatifs qui questionnent la base de texte, remet en question la distance informationnelle entre l'administration publique (ici locale) et les citoyens, censés « participer » à la vie démocratique. Cette impossible participation, largement discutée et dénoncée sur le plan de la conception même de la démocratie dans nos sociétés modernes, s'inscrit dans les pratiques langagières : la communication de l'information municipale en est un cas.

Au Québec, plus encore peut-être qu'ailleurs, le palier municipal, sous couvert d'autonomie implicite (Loi constitutionnelle de 1867, i.e. décentralisation) malmenée par des tensions entre le provincial et le fédéral pour le financement des municipalités « est une manière d'imposer un peu de sa volonté aux autres paliers de gouvernement, c'est-à-dire de refléter autant que faire se peut ce que le citoyen lui demande à l'intérieur des champs d'activités qui relèvent de ses compétences. Elle réfère à un contrôle local plus sensible aux besoins des citoyens, à une plus grande flexibilité de gestion, à une meilleure adéquation des ressources disponibles et des besoins des individus » (Rivest, 2009 : 40). Si les moyens financiers de cette autonomie ont été historiquement bafoués, force est de constater que les discours de l'information publique municipale créent eux aussi un fossé plutôt qu'un rapprochement entre la « gouvernance » locale et les demandes des citoyens. Est-ce à dire que le discours administratif public des villes entérine les obstacles financiers et politiques à l'exercice citoyen, déléguant au langage l'odieux d'ancrer la distance sociocognitive entre le peuple et l'institution? 


\section{Bibliographie}

Authier-Revuz Jacqueline (1995), Ces mots qui ne vont pas de soi : boucles réflexives et non-coïncidences du dire, Paris, Larousse.

Bikialo Stéphane (2018), Discours hors-normes et discours littéraire : ce qu'en disent la ponctuation et les genres de discours (ici même).

Bonnafous Simone et Malika Temmar (dir.) (2007), Analyse du discours et sciences humaines et sociales, Paris, Ophrys.

Borel Marie-Jeanne, Grize Jean-Blaise et Miéville Denis (1983, 2éd. 1992), Essai de logique naturelle, Berne, P. Lang.

Centre de Linguistique Appliquée de Besançon, Rui Blandine, Collette Karine et al. (2002), Guide pratique de la rédaction administrative, Paris, Éditions du ministère de la Fonction publique et de la réforme de l'État.

Clerc Isabelle (dir.), (2003). Simplification des lettres de l'Administration adressées au grand public, Québec.

Collette Karine (2007a), "La concurrence discursive : analyse des logiques de discours dans la communication épistolaire adressée par l'administration publique aux usagers », Communication : information, médias, théorie, pratique, vol. 25 (2), 107-130.

Collette Karine (2007b), La Dimension sociale dans les pratiques de lecture en formation : approche sociocognitive et implications didactiques, Chaire Normand Maurice, Trois-Rivières, UQTR, n 5.

Collette Karine (2008 a) «Parcours interprétatifs sur des courriers administratifs : lieu et place de l'extratextuel », A.-M. Houdebine et al. (dir.), Les Aventures de l'interprétation, Actes du colloque international «Sémiologie 2005 », Paris, Laboratoire DynaLang, 101-110.

Collette Karine (2008b), "Qualité de la relation entre administration et usagers : la part informationnelle de l'asymétrie. Interprétation d'usagers et approche macro-discursive de la marge rédactionnelle », Technostyle, vol. 22, $n^{\circ} 1,19-38$.

Collette Karine (2008 c), « Construire la relation à l'usager dans un contexte sociopolitique : exemple de la rédaction administrative », I. Clerc et C. Beaudet (dir.), Langue, médiation et efficacité communicationnelle, Québec, PUL, 81-107.

Collette Karine (2008d) «L'écriture alternative : à la recherche d'ingrédients pour une écriture citoyenne », Pré-actes du colloque « De la France au Québec. L'écriture dans tous ses états », Poitiers.

Collette Karine (2008e), «L'interdiscursivité des écrits “autour” du conseil municipal », L. S. Florea, C. Papghi, L. Pop et A. Curea (dir.), Directions actuelles en linguistique du texte, actes du colloque international Le texte: modèles, méthodes, perspectives, II, C. Napoca, C. Cartii de Stiinta, 303-312.

Collette Karine (2009), «Pour une approche empirique du lecteur-citoyen », G. Holtzer, D. Lebaud (dir.), Parcours, traces. Autour du texte et des langues. Mélanges offerts en hommage à Marc Souchon, Besançon, PUFC, 93-104.

Collette Karine et Steuckardt Agnès (2016), "Présentation : Discours hors-normes, constructions sociales », Signes, discours et sociétés n 16, http://www.revue-signes.info/sommaire.php?id=4640

Grize Jean-Blaise (1990), Logique et langage, Paris, Ophrys. 
Grize Jean-Blaise (1996), Logique naturelle et communications, Paris, PUF.

Halbwachs Maurice (1952), Les Cadres sociaux de la mémoire, Paris, PUF.

Kintsch Walter (1988), "The Role of Knowledge in Discourse Processing: A Construction-Integration Model", Psychological Review, 95, 163-182.

Kintsch Walter (1998), Comprehension: a paradigm for cognition, New York, Cambridge University Press.

Lahire Bernard (2001), L'Homme pluriel. Les ressorts de l'action, Paris, Nathan.

Martin-Lagardette Jean-Luc (2005,), Le Guide de l'écriture journalistique, 6éd., Paris, La Découverte.

Rivest Martin (2009), Persister persuade. Union des municipalités du Québec. 90 ans d’histoire 1919-2009, Québec, UMQ.

Sperber Dan et Deirdre Wilson (1989), La Pertinence, communication et cognition, Paris, Les Éditions de Minuit.

Tapiero Isabelle (1992), Traitement cognitif du texte narratif et expositif et connexionnisme : expérimentation et simulation. Thèse de doctorat nouveau régime, Université Paris VIII.

Van Dijk Teun A. et Kintsch Walter (1983), Strategies of discourse comprehension, New York, Academic Press. Windisch Ulli (1982), Pensée sociale, langage en usage et logiques autres, Lausanne, L’Âge d'Homme.

Windisch Ulli (1985), Le Raisonnement et le parler quotidiens, Lausanne, L’Âge d’Homme.

Windisch Ulli (1990), Le Prêt-à-penser, les formes de la communication et de l'argumentation quotidiennes, Lausanne, Paris, L’Âge d'Homme. 